\title{
Long Dominating Cycles and Paths in Graphs with Large Neighborhood Unions
}

H. J. Broersma H. J. Veldman DEPARTMENT OF APPLIED MATHEMATICS UNIVERSITY OF TWENTE P. O. BOX 217 7500 AE ENSCHEDE, THE NETHERLANDS

\section{ABSTRACT}

Let $G$ be a graph of order $n$ and define $N C(G)=\min \{|N(u) \cup N(v)|$ $\mid u v \notin E(G)\}$. A cycle $C$ of $G$ is called a dominating cycle or D-cycle if $\eta G)-(C C)$ is an independent set. AD-path is defined analogously. The following result is proved: if $G$ is 2-connected and contains a $D$-cycle, then $G$ contains a $D$-cycle of length at least $\min \{n, 2 N C(G)\}$ unless $G$ is the Petersen graph. By combining this result with a known sufficient condition for the existence of a $D$-cycle, a common generalization of Ore's Theorem and several recent "neighborhood union results" is obtained. An analogous result on long $D$-paths is also established.

\section{TERMINOLOGY AND NOTATIONS}

We use [3] for terminology and notations not defined here, and consider simple graphs only. Throughout, let $G$ be a graph of order $n$.

If $G$ has a Hamilton cycle (a cycle containing every vertex of $G$ ), then $G$ is called hamiltonian. $G$ is traceable if $G$ has a Hamilton path (a path containing every vertex of $G$ ). A cycle $C$ of $G$ is called a dominating cycle, or briefly D-cycle, if $V(G)-V(C)$ is an independent set of vertices in $G$. A dominating path or D-path is analogously defined. Two edges $e_{1}$ and $e_{2}$ of $G$ are called remote if they are nonadjacent, and there is no edge of $G$ joining an end of $e_{1}$ and one of $e_{2}$. The degree of an edge $u v$ of $G$ is the number of vertices in $V(G)-\{u, v\}$ adjacent to at least one of the vertices $u$ and $v$. 
The length of a longest cycle in $G$ is denoted by $c(G)$, the order of a longest path by $p(G)$, the number of vertices in a maximum independent set by $\alpha(G)$, and the set of vertices adjacent to a vertex $v$ by $N(v)$. We denote by $\sigma_{k}(G)$ the minimum value of the degree-sum of any $k$ pairwise nonadjacent vertices; if $k>\alpha(G)$, we set $\sigma_{k}(G)=k(n-1)$. Instead of $\sigma_{1}(G)$ we use the more common notation $\delta(G)$. We denote by $\sigma_{k}^{\prime}(G)$ the minimum value of the degree-sum of any $k$ pairwise remote edges; if $G$ does not contain $k$ pairwise remote edges, then $\sigma_{k}^{\prime}(G)=k(n-2)$. If $G$ is noncomplete, then $N C(G)$ denotes $\min \{|N(u) \cup N(v)| \mid u v \notin E(G), u \neq v\}$; if $G$ is complete, we set $N C(G)=n-1$. If $|E(G)|>0$, then $N C^{\prime}(G)$ denotes $\min \{|N(u) \cup N(v)|$ $\mid u v \in E(G)\}$; otherwise, $N C^{\prime}(G)=0$. By $N C^{\prime \prime}(G)$ we denote $\min \{\mid N(u) \cup$ $N(v)|| u, v \in V(G), u \neq v\}$. If no ambiguity can arise, we sometimes write $\alpha$ instead of $\alpha(G), \sigma_{k}$ instead of $\sigma_{k}(G)$, etc.

We now define two special classes of graphs. For $n \geq 5$, the graph $G_{n}$ is defined as the join of $K_{2}$ and the graph of order $n-2$ consisting of three disjoint complete graphs, the orders of which pairwise differ by at most one. For $n \geq 4$, the graph $H_{n}$ is obtained from $G_{n+1}$ by deleting a vertex of degree $n$.

\section{MAIN RESULT AND CONSEQUENCES}

A slightly stronger version of the following result was recently established.

Theorem 1 [1]. If $G$ is 2 -connected and $\sigma_{3}(G) \geq n+2$, then $c(G) \geq$ $\min \{n, 2 N C(G)\}$.

It was shown in [1] that Theorem 1 is a common generalization of results in [5], [6], and [7].

A key ingredient in the proof of Theorem 1 is the following result of Bondy:

Theorem 2 [2]. If $G$ is 2 -connected and $\sigma_{3}(G) \geq n+2$, then every longest cycle of $G$ is a $D$-cycle.

By the role of Theorem 2 in the proof of Theorem 1 we were led to investigate whether the conclusion of Theorem 1 still holds if $G$ is only required to be 2-connected and to have a $D$-cycle. Our main result is as follows.

Theorem 3. If $G$ is 2 -connected and contains a $D$-cycle, then $G$ contains a $D$-cycle of length at least $\min \{n, 2 N C(G)\}$ unless $G$ is the Petersen graph.

Note that $c(G)=2 N C(G)-1$ if $G$ is the Petersen graph.

The proof of Theorem 3 is postponed to Section 3 .

The conclusion of Theorem 3 cannot be strengthened, as shown by complete bipartite graphs: for $2 \leq r \leq s$ we have $c\left(K_{r, s}\right)=2 r=2 N C\left(K_{r, s}\right)$. 
Furthermore, the requirement that $G$ contain a $D$-cycle, cannot be omitted: for $n \geq 8$ the graph $G_{n}$ contains no $D$-cycle, while $c\left(G_{n}\right)=N C\left(G_{n}\right)+2$ if $n \equiv 2(\bmod 3)$ and $c\left(G_{n}\right)=N C\left(G_{n}\right)+3$ otherwise.

By combining Theorem 3 with Theorem 2 we obtain Theorem 1.

The following condition for the existence of a $D$-cycle occurs in [8].

Theorem 4 [8]. If $G$ is 2 -connected and $\sigma_{3}^{\prime}(G) \geq n-1$, then $G$ contains a $D$-cycle.

It was observed in [8] that the hypothesis of Theorem 4 is weaker than the hypothesis of Theorem 2. (Note that, on the other hand, the conclusion of Theorem 4 is weaker than the conclusion of Theorem 2.) Thus by combining Theorem 3 with Theorem 4 we obtain a result that is more general than Theorem 1.

Theorem 5. If $G$ is 2-connected and $\sigma_{3}^{\prime}(G) \geq n-1$, then $c(G) \geq$ $\min \{n, 2 N C(G)\}$ unless $G$ is the Petersen graph.

Corollary 6. If $G$ is 2-connected and $N C^{\prime}(G) \geq \frac{1}{3}(n+5)$, then $c(G) \geq$ $\min \{n, 2 N C(G)\}$ unless $G$ is the Petersen graph.

Proof. If $G$ is 2-connected and $N C^{\prime}(G) \geq \frac{1}{3}(n+5)$, then

$$
\sigma_{3}^{\prime}(G) \geq 3 \sigma_{1}^{\prime}(G)=3\left(N C^{\prime}(G)-2\right) \geq n-1 .
$$

Corollary 6 complements and partially improves the following result, since clearly $N C^{\prime \prime}(G) \leq \min \left\{N C(G), N C^{\prime}(G)\right\}$ :

Theorem 7 [4]. If $G$ is 2-connected and $N C^{\prime \prime}(G) \leq \frac{1}{2} n$, then $c(G) \geq$ $2 N C^{\prime \prime}(G)-2$. For $N C^{\prime \prime}(G) \leq \frac{1}{3}(n+4)$, the result is sharp in the sense that longer cycles are not implied by the conditions.

An immediate consequence of Corollary 6 is the following:

Corollary 8. If $G$ is 2 -connected, $N C^{\prime}(G) \geq \frac{1}{3}(n+5)$, and $N C(G) \geq \frac{1}{2} n$, then $G$ is hamiltonian unless $G$ is the Petersen graph.

Corollary 8 improves the following result.

Theorem 9 [4]. If $G$ is 2 -connected and $N C^{\prime \prime}(G) \geq \frac{1}{2} n$, then, for $n$ sufficiently large, $G$ is hamiltonian.

Theorem 3 has the following analogue: 
Theorem 10. If $G$ is connected and contains a $D$-path, then $G$ contains a $D$-path of order at least $\min \{n, 2 N C(G)+1\}$.

Proof. Apply Theorem 3 to the join of $G$ and $K_{1}$.

Again the complete bipartite graphs show that the conclusion of Theorem 10 cannot be strengthened. Furthermore, the requirement that $G$ contain a $D$-path cannot be omitted, as shown by the graph $H_{n}$ for $n \geq 7$.

Theorem 10 admits corollaries similar to those of Theorem 3 .

\section{PROOF OF THE MAIN RESULT}

Throughout this section we assume that

$-G$ is 2-connected and nonhamiltonian,

$-C$ is a longest $D$-cycle of $G$ for which $\max \{d(v) \mid v \in V(G)-V(C)\}$ is as large as possible,

$-|V(C)| \leq 2 N C-1$.

We first introduce some additional notations. By $\vec{C}$ we denote the cycle $C$ with a given orientation. Let $u, v \in V(C)$. By $u \vec{C} v$ we denote the consecutive vertices on $C$ from $u$ to $v$ in the direction specified by $\vec{C}_{\vec{C}}$ The same vertices, in reverse order, are given by $v \overleftrightarrow{C} u$. We will consider $u \vec{C} v$ and $v \overleftrightarrow{C} u$ both as paths and as vertex sets. We use $u^{+}$to denote the successor of $u$ on $\vec{C}$ and $u^{-}$to denote its predecessor. We write $u^{++}$instead of $\left(u^{+}\right)^{+}$and $u^{--}$ instead of $\left(u^{-}\right)^{-}$. If $S \subseteq V(C)$, then $S^{+}=\left\{x^{+} \mid x \in S\right\}$ and $S^{-}=\left\{x^{-} \mid x \in S\right\}$. We write $u v \in P_{C}(G)$ if $u$ and $v$ are connected by a path of length at least 2 that is internally disjoint from $C$.

Before proving Theorem 3 we establish a number of lemmas, the first four of which have become so standard in hamiltonian graph theory that we omit their proofs.

Lemma 11. If $v \in V(C)$, then $v v^{+} \notin P_{C}(G)$.

Lemma 12. If $x \in V(G)-V(C)$ and $v_{1}, v_{2} \in N(x)$, then $v_{1}^{+} v_{2}^{+}, v_{1}^{-} v_{2}^{-} \notin$ $E(G) \cup P_{C}(G)$.

Lemma 13. Let $x \in V(G)-V(C), v_{1}, v_{2} \in N(x)$ and $v \in v_{2}^{+} \vec{C} v_{1}^{-}$. If $v_{1}^{+} v \in E(G) \cup P_{C}(G)$, then $v_{2}^{+} v^{+} \notin E(G) \cup P_{C}(G)$. If $v_{2}^{-} v \in E(G) \cup$ $P_{C}(G)$, then $v_{1}^{-} v^{-} \notin E(G) \cup P_{C}(G)$.

Lemma 14. Let $x \in V(G)-V(C), v_{1}, v_{2} \in N(x)$ and $v \in v_{2}^{+} \vec{C} v_{1}^{-}$. If $v_{1}^{+} v \in \mathrm{E}(G) \cup P_{C}(G)$, then $v_{2}^{-} v^{-}, v_{2}^{-} v^{+} \notin E(G) \cup P_{C}(G)$. 
Lemma 15. Let $x \in V(G)-V(C)$ and $v_{1}, v_{2} \in N(x)$. If $v_{1}^{+} v_{2}^{++} \in P_{C}(G)$, then $N\left(v_{2}^{+}\right) \cap(V(G)-(V(C) \cup\{x\})) \neq \varnothing$. If $v_{1}^{-} v_{2}^{--} \in P_{C}(G)$, then $N\left(v_{2}^{-}\right) \cap$ $(V(G)-(V(C) \cup\{x\})) \neq \varnothing$.

Proof. By symmetry, we need only prove the first part of the lemma. Suppose $v_{1}^{+} v_{2}^{++} \in P_{C}(G)$ and $N\left(v_{2}^{+}\right) \cap(V(G)-(V(C) \cup\{x\}))=\varnothing$. Let $v_{1}^{+} x_{1} \cdots x_{r} v_{2}^{++}$be a $\left(v_{1}^{+}, v_{2}^{++}\right)$-path that is internally disjoint from $C(r \geq 1)$. By Lemma 11, $x \notin\left\{x_{1}, \cdots, x_{r}\right\}$. Now the cycle $v_{1} x v_{2} \overleftrightarrow{C} v_{1}^{+} x_{1} \cdots x_{r} v_{2}^{++} \vec{C} v_{1}$ is a $D$-cycle longer than $C$, a contradiction.

Lemma 16. Let $x_{1}, x_{2} \in V(G)-V(C), A_{1}=N\left(x_{1}\right), A_{2}=N\left(x_{2}\right)$ and $A=$ $A_{1} \cup A_{2}$. Then $|A|=N C$ and either $\left|A \cap A^{+}\right|=1$ and $A \cup A^{+}=V(C)$ or $\left|A \cap A^{+}\right|=2$ and $\left|A \cup A^{+}\right| \geq|V(C)|-1$.

Proof. By Lemma 12, $\left|A_{1} \cap A_{2}^{+}\right| \leq 1$ and $\left|A_{2} \cap A_{1}^{+}\right| \leq 1$. Using Lemma 11 we conclude that $\left|A \cap A^{+}\right| \leq 2$. Hence

$$
2 N C-1 \geq|V(C)| \geq\left|A \cup A^{+}\right|=|A|+\left|A^{+}\right|-\left|A \cap A^{+}\right| \geq 2|A|-2,
$$

implying that $|A| \leq N C$ and hence $|A|=N C$. The rest of the lemma also follows.

Lemma 17. Let $x \in V(G)-V(C)$ and $y \in V(C)$. Then $x y^{+} \notin E(G)$ or $x y^{-} \notin E(G)$.

Proof. Suppose $x y^{+}, x y^{-} \in E(G)$. If $N(y) \cap(V(G)-(V(C) \cup\{x\}))=\varnothing$, then $N(x) \cup N(y) \subseteq V(C)$ and, by Lemmas 11, 12, and 14, $v^{+} \notin N(x) \cup$ $N(y)$ whenever $v \in N(x) \cup N(y)$, implying that $|N(x) \cup N(y)| \leq \frac{1}{2}|V(C)|<$ $N C$, a contradiction. Hence $y$ has a neighbor $x_{1}$ in $V(G)-(V(C) \cup\{x\})$. Set $A=N(x) \cup N\left(x_{1}\right)$. By Lemmas 11 and 12, $y^{++}, y^{--} \notin A$. From Lemma 16 we conclude that $y^{+++} \in A$ or $y^{---} \in A$. Assume without loss of generality that $y^{+++} \in A$ and set $w=y^{+++}$. Then $w^{-}$has a neighbor $x_{2}$ in $V(G)-(V(C) \cup\{x\})$ : assuming the contrary, we obtain a contradiction as in the beginning of the proof if $w \in N(x)$, while we contradict Lemma 15 if $w \in N\left(x_{1}\right)$. By Lemma $12, x_{2} \neq x_{1}$.

We claim that $N\left(x_{2}\right) \cap\left(V(C)-\left(A \cup A^{+}\right)\right)=\varnothing$. This is clear if $V(C)=A \cup A^{+}$. Otherwise, by Lemma 16, $V(C)-\left(A \cup A^{+}\right)$contains a unique vertex $z$ and $z^{+} \in A$. If $w, z^{+} \in N(x)$, then $x_{2} z \notin E(G)$ by Lemma 12. If $w \in N(x)$ and $z^{+} \in N\left(x_{1}\right)$, then $x_{2} z \notin E(G)$ by Lemma 14. If $w \in N\left(x_{1}\right)$ and $z^{+} \in N(x)$, then $x_{2} z \notin E(G)$ by Lemma 13. Finally, if $w, z^{+} \in N\left(x_{1}\right)$, then $x_{2} z \notin E(G)$ by Lemma 12 . Hence, indeed, $N\left(x_{2}\right) \cap$ $\left(V(C)-\left(A \cup A^{+}\right)\right)=\varnothing$.

By Lemmas 12 and 13, $N\left(x_{2}\right) \cap\left(A^{+}-\left\{w^{-}\right\}\right)=\varnothing$. Now if $w \notin N\left(x_{1}\right)$, it follows that $N\left(x_{1}\right) \cup N\left(x_{2}\right) \subseteq\left(A \cup\left\{w^{-}\right\}\right)-\left\{y^{+}, w\right\}$, whence $\mid N\left(x_{1}\right) \cup$ 
$N\left(x_{2}\right)|<| A \mid=N C$, a contradiction. If $w \in N\left(x_{1}\right)$, then $N\left(x_{1}\right) \cup$ $N\left(x_{2}\right) \subseteq\left(A \cup\left\{w^{-}\right\}\right)-\left\{y^{+}, y^{-}\right\}$, and we again obtain a contradiction.

Lemma 18. If $x_{1}, x_{2} \in V(G)-V(C)$, then $N\left(x_{1}\right) \cap N\left(x_{2}\right)=\varnothing$.

Proof. Suppose $N\left(x_{1}\right) \cap N\left(x_{2}\right)$ contains a vertex $y$. From Lemma 16 we conclude that $y^{++}$or $y^{--}$is in $N\left(x_{1}\right) \cup N\left(x_{2}\right)$. In either case we contradict Lemma 17.

Proof of Theorem 3. We distinguish two main cases and a number of subcases, in each of which we either reach contradictions with the assumptions at the beginning of this section or the conclusion that $G$ is the Petersen graph.

Case 1. $|V(G)-V(C)| \geq 2$.

If $|V(G)-V(C)| \geq 4$, then by Lemma $18 V(G)-V(C)$ contains two vertices $z_{1}, z_{2}$ with $\left|N\left(z_{1}\right) \cup N\left(z_{2}\right)\right| \leq \frac{1}{2}|V(C)|<N C$, a contradiction. Hence $|V(G)-V(C)| \leq 3$.

Let $x_{1}$ and $x_{2}$ be two vertices in $V(G)-V(C)$. Set $A_{i}=N\left(x_{i}\right)(i=1,2)$ and $A=A_{1} \cup A_{2}$. By Lemma 16 there are two possibilities.

Case 1.1. $\left|A \cap A^{+}\right|=1$ and $A \cup A^{+}=V(C)$.

Let $y$ be the unique vertex in $A \cap A^{+}$. Assume without loss of generality that $x_{1} y^{-}, x_{2} y \in E(G)$. Set $w=y^{++}, z=w^{++}$. By Lemma $17, w \in A_{1}$ and $z \in A_{2}$. By Lemma $15, w^{+}$has a neighbor $x_{3}$ in $V(G)-\left(V(C) \cup\left\{x_{1}, x_{2}\right\}\right)$. Since $G$ is 2-connected, $x_{3}$ has a neighbor $v$ on $V(C)$ with $v \neq w^{+}$. By Lemmas 17 and $18, v \in z^{++} \vec{C} y^{--}$and $v \notin A$. Hence $v \in A^{+}$. If $v^{-} \in A_{1}$, then we contradict Lemma 12 (with $x=x_{1}$ ). If $v^{-} \in A_{2}$, then we contradict Lemma 13 (with $x=x_{1}$ ).

Case 1.2. $\left|A \cap A^{+}\right|=2$ and $\left|A \cup A^{+}\right| \geq|V(C)|-1$.

Set $A \cap A^{+}=\left\{y_{1}, y_{2}\right\}$. Assume without loss of generality that $y_{1}^{-} \in$ $N\left(x_{1}\right), y_{1} \in N\left(x_{2}\right)$. Using Lemma 12 we conclude that $y_{2} \in N\left(x_{1}\right), y_{2}^{-} \in$ $N\left(x_{2}\right)$. Since $\left|V(C)-\left(A \cup A^{+}\right)\right| \leq 1$, we may assume without loss of generality that $V(C)-\left(A \cup A^{+}\right) \subseteq y_{1} \vec{C} y_{2}^{-}$. Set $w=y_{2}^{++}, z=w^{++}$. Since $y_{2} \vec{C} y_{1}^{-} \subseteq A \cup A^{+}, w, z \in A$. By Lemma $17, w \in A_{2}$ and $z \in A_{1}$. By Lemma $15, y_{2}^{+}$has a neighbor $x_{3}$ in $V(G)-\left(V(C) \cup\left\{x_{1}, x_{2}\right\}\right)$ while $w^{+}$has a neighbor $x_{4}$ in $V(G)-\left(V(C) \cup\left\{x_{1}, x_{2}\right\}\right)$. Since $|V(G)-V(C)| \leq 3$, $x_{3}=x_{4}$. But then we contradict Lemma 17 (with $x=x_{3}$ ).

Case 2. $|V(G)-V(C)|=1$

Since we have assumed that $|V(C)| \leq 2 N C-1$, we have $N C \geq \frac{1}{2} n$. Let $x$ be the unique vertex in $V(G)-V(C)$ and $v_{1}, \ldots, v_{k}$ the neighbors of $x$, oc- 
curring on $\vec{C}$ in the order of their indices. For $i=1, \ldots, k$, set $u_{i}=v_{i}^{+}$, $w_{i}=v_{i+1}^{-}$and $T_{i}=u_{i} \vec{C} w_{i}$ (indices $\bmod k$ ). We call the sets $T_{1}, \ldots, T_{k}$ segments of $C ; T_{i}$ is a t-segment if $\left|T_{i}\right|=t(i=1, \ldots, k)$. We set $T=\cup_{i=1}^{k} T_{i}$. For a vertex $v$ of $G$ and an integer $i \in\{1, \ldots, k\}$, we denote $N(v) \cap T_{i}$ by $N_{\mathrm{i}}(v)$; by $N_{T}(v)$ we denote $\cup_{i=1}^{k} N_{i}(v)$.

By Lemma 17, $C$ contains no 1-segments. Two possibilities remain.

Case 2.1. C contains a 2-segment.

Assume without loss of generality that $T_{1}$ is a 2 -segment. Define the function $f: V(G) \rightarrow\{0,1,2\}$ by $f(v)=\left|N(v) \cap\left\{u_{1}, w_{1}\right\}\right|$. From Lemmas 12 and 14 we deduce that

$$
f\left(u_{i}\right)+f\left(w_{i}\right) \leq 1 \text { for } i=2, \ldots, k .
$$

Lemma 14 also implies that

$$
\text { if } v \in u_{2} \vec{C} w_{k} \text { and } f(v)=2 \text {, then } f\left(v^{-}\right)=f\left(v^{+}\right)=0 .
$$

From (1) and (2) we conclude that

$$
\left|N_{i}\left(u_{1}\right)\right|+\left|N_{i}\left(w_{1}\right)\right|=\sum_{v \in T_{i}} f(v) \leq\left|T_{i}\right|-1 \quad \text { for } i=2, \ldots, k
$$

whence

$$
\left|N_{T}\left(u_{1}\right)\right|+\left|N_{T}\left(w_{1}\right)\right| \leq 2+\sum_{i=2}^{k}\left(\left|T_{i}\right|-1\right)=|V(C)|-2 k+1 .
$$

Assuming without loss of generality that $\left|N_{T}\left(u_{1}\right)\right| \leq\left|N_{T}\left(w_{1}\right)\right|$ we thus have

$$
\left|N_{T}\left(u_{1}\right)\right| \leq \frac{1}{2}(|V(C)|+1)-k=\frac{1}{2} n-k,
$$

implying that

$$
\frac{1}{2} n \leq N C \leq\left|N(x) \cup N\left(u_{1}\right)\right|=k+\left|N_{T}\left(u_{1}\right)\right| \leq \frac{1}{2} n .
$$

Since all inequalities in (4) are in fact equalities, (3) also holds with equality:

$$
\sum_{v \in T_{i}} f(v)=\left|T_{i}\right|-1 \quad \text { for } i=2, \ldots, k
$$

We now show that

$$
f\left(u_{i}^{+}\right) \neq 2 \text { and } f\left(w_{i}^{-}\right) \neq 2 \text { for } i=2, \ldots, k .
$$


Suppose, e.g., $f\left(u_{i}^{+}\right)=2$ for some $i \in\{2, \ldots, k\}$. If $v \in u_{i}^{+} \vec{C} w_{k}$ and $u_{i} v, u_{i} v^{+} \in E(G)$, then the cycle $v_{1} x v_{i} \stackrel{C}{C} u_{1} u_{i}^{+} \vec{C} v u_{i} v^{+} \vec{C} v_{1}$ contradicts the choice of $C_{\dot{+}}$ If $v \in u_{1} \vec{C} w_{i-1}$ and $u_{i} v, u_{i} v^{+} \in E(G)$, then the cycle $v_{1} x v_{i}{ }_{C} v^{+} u_{i} v C u_{1} u_{i}^{+} \vec{C} v_{1}$ contradicts the choice of $C$. Together with Lemmas 11-14 these observations show that $v^{+} \notin N(x) \cup N\left(u_{i}\right)$ whenever $v \in N(x) \cup N\left(u_{i}\right)$. But then $\left|N(x) \cup N\left(u_{i}\right)\right| \leq \frac{1}{2}|V(C)|<\frac{1}{2} n \leq N C$. This contradiction establishes (6).

In view of (6), (5) holds only if

$$
\begin{aligned}
& \text { for } i=2, \ldots, k, \text { either } f\left(u_{i}\right)=0 \text { and } f(v)=1 \text { for every } \\
& v \in T_{i}-\left\{u_{i}\right\} \text { or } f\left(w_{i}\right)=0 \text { and } f(v)=1 \text { for every } v \in T_{i}-\left\{w_{i}\right\} .
\end{aligned}
$$

If, for some $i \in\{2, \ldots, k\}, N_{i}\left(u_{1}\right) \neq \varnothing$ and $N_{i}\left(w_{1}\right) \neq \varnothing$, then by (7) $T_{i}$ contains a vertex $v$ such that either $u_{1} v, w_{1} v^{+} \in E(G)$ or $u_{1} v^{+}, w_{1} v \in E(G)$, contradicting Lemma 14 . Hence

$$
\text { for } i=2, \ldots, k \text {, either } N_{i}\left(u_{1}\right)=\varnothing \text { or } N_{i}\left(w_{1}\right)=\varnothing \text {. }
$$

Combining (7) and (8) and using Lemma 12 we conclude that

$$
\begin{aligned}
& \text { for } i=2, \ldots, k \text {, either } f\left(u_{i}\right)=0 \text { and } u_{1} v \in E(G) \text { for every } v \in \\
& T_{i}-\left\{u_{i}\right\} \text { or } f\left(w_{i}\right)=0 \text { and } w_{1} v \in E(G) \text { for every } v \in T_{i}-\left\{w_{i}\right\} .
\end{aligned}
$$

We now show that all segments of $C$ are 2-segments. Suppose there exist integers $r$ and $t$ with $2 \leq r \leq k$ and $t \geq 3$ such that $T_{r}$ is a $t$-segment. In view of (9) we may assume, without loss of generality, that $f\left(u_{r}\right)=0$ and $u_{1} v \in E(G)$ for every $v \in T_{r}-\left\{u_{r}\right\}$. By (9) and Lemma $14, N_{i}\left(w_{1}\right)=\varnothing$ for $i=2, \ldots, r$. If $r<k$, then $N_{i}\left(w_{1}\right)=\varnothing$ for $i=r+1, \ldots, k$ also, otherwise $w_{1} u_{s} \in \mathrm{E}(G)$ for some $s \in\{r+1, \ldots, k\}$ and the cycle $v_{1} x v_{s} \stackrel{\leftarrow}{C} w_{r} u_{1} w_{r}^{-} \stackrel{\leftarrow}{C}$ $w_{1} u_{s} \vec{C} v_{1}$ contradicts the choice of $C$. It follows that

$$
\left|N(x) \cup N\left(w_{1}\right)\right|=k+\left|N_{T}\left(w_{1}\right)\right|=k+1 \leq \frac{1}{3}(n-2)+1<\frac{1}{2} n .
$$

This contradiction shows that $C$ indeed contains 2-segments only, implying that $n=3 k+1$.

Set $m=\max \left\{i \mid u_{1} w_{i} \in E(G)\right\}$. Then, by (9) and Lemma $14, u_{1} w_{i} \in E(G)$ for $1 \leq i \leq m$, while $w_{1} u_{j} \in E(G)$ for $j=1$ and $m+1 \leq j \leq k$. If $m<\frac{1}{2}(k+1)$, then

$$
\left|N(x) \cup N\left(u_{1}\right)\right|=k+\left|N_{T}\left(u_{1}\right)\right|=k+m<\frac{1}{2}(3 k+1)=\frac{1}{2} n,
$$

a contradiction. If $m>\frac{1}{2}(k+1)$, then

$$
\left|N(x) \cup N\left(w_{1}\right)\right|=k+\left|N_{T}\left(w_{1}\right)\right|=k+k-m+1<\frac{1}{2}(3 k+1)=\frac{1}{2} n,
$$

again a contradiction. Hence $m=\frac{1}{2}(k+1)$ and $k$ is odd. 
We have shown that $u_{1} w_{i} \in E(G)$ for $1 \leq i \leq m$. By the same token, $u_{k} w_{i} \in E(G)$ for $1 \leq i \leq m-1$. Now by Lemma $13, u_{1} v_{i} \notin E(G)$ for $i=2, \ldots, m$. By Lemma $14, u_{1} v_{i} \notin E(G)$ for $i=m+1, \ldots, k$. Hence $N\left(u_{1}\right) \subseteq\left\{w_{1}, \ldots, w_{m}, v_{1}\right\}$ and, similarly, $N\left(u_{2}\right) \subseteq\left\{w_{2}, \ldots, w_{m+1}, v_{2}\right\}$. Thus

$$
\frac{1}{2} n \leq\left|N\left(u_{1}\right) \cup N\left(u_{2}\right)\right| \leq m+3=\frac{1}{2}(k+1)+3=\frac{1}{2} n-k+3 .
$$

It follows that $k=3$ and $N\left(u_{1}\right)=\left\{w_{1}, w_{2}, v_{1}\right\}$. An argument of symmetry gives us $N\left(u_{i}\right)$ and $N\left(w_{i}\right)$ for each $i \in\{1,2,3\}$. We conclude that the Petersen graph is a spanning subgraph of $G$. Since the Petersen graph is a maximal nonhamiltonian graph, $G$ itself must be the Petersen graph.

Case 2.2. $C$ contains no 2-segments.

Then $d(x) \leq \frac{1}{4}|V(C)|<\frac{1}{4} n$. If $v \in T$, then $|N(x) \cup N(v)| \geq \frac{1}{2} n$, implying that $d(v)>\frac{1}{4} n$. From the maximality of $\max \{\mathrm{d}(v) \mid v \in V(G)-V(C)\}$, we conclude that

if $v \in T$, then $G$ contains no cycle with vertex set $V(G)-\{v\}$.

For $i=1,2$, let $y_{i}$ be the first vertex of $T_{i}$ such that $y_{i} v_{i}^{-} \notin E(G)$. Set $Y_{11}=N_{1}\left(y_{1}\right), Y_{12}=\left(N_{1}\left(y_{2}\right)\right)^{+}, Y_{21}=\left(N_{2}\left(y_{1}\right)\right)^{+}, Y_{22}=N_{2}\left(y_{2}\right)$, and for $i=$ $3, \ldots, k, Y_{i 1}=N_{i}\left(y_{1}\right), Y_{i 2}=\left(N_{i}\left(y_{2}\right)\right)^{-}$. By a variation of Lemma 12 we have

$y_{1} v \notin E(G)$ for $v \in u_{2} \vec{C} y_{2}, y_{2} v \notin E(G)$ for $v \in u_{1} \vec{C} y_{1}$, and $y_{i} u_{j} \notin E(G)$ for $i=1,2$ and $j=3, \ldots, k$.

(If, e.g., $y_{1} y_{2} \in E(G)$, then the cycle $v_{1} x v_{2} \vec{C} y_{2}^{-} w_{1} \overleftarrow{C} y_{1} y_{2} \vec{C} w_{k} y_{1}^{-} \overleftarrow{C} v_{1}$ contradicts the choice of $C$ ). From (11) and a similar variation of Lemma 13 we deduce that $Y_{i 1} \cap Y_{i 2}=\varnothing(i=1, \ldots, k)$. By (11) and the way $y_{2}$ was chosen, $Y_{11} \cup Y_{12} \subseteq T_{1}-\left\{y_{1}\right\}$. By (11) and (10) (with $\left.v=u_{i}\right), Y_{i 1} \cup Y_{i 2} \subseteq T_{i}-\left\{u_{i}\right\}$ $(i=3, \ldots, k-1)$. If $k \geq 3$, then $Y_{21} \cup Y_{22} \subseteq\left(T_{2}-\left\{y_{2}\right\}\right) \cup\left\{v_{3}\right\}$ (by (11)) and $Y_{k 1} \cup Y_{k 2} \subseteq T_{k}-\left\{u_{k}, w_{k}\right\}$ (by (11), (10), and the way $y_{1}$ was chosen). If $k=2$, then the choice of $y_{1}$ implies $Y_{21} \cup Y_{22} \subseteq T_{2}-\left\{y_{2}\right\}$. In both cases we conclude that

$$
\begin{aligned}
\left|N_{T}\left(y_{1}\right)\right|+\left|N_{T}\left(y_{2}\right)\right| & =\sum_{i=1}^{k}\left(\left|N_{i}\left(y_{1}\right)\right|+\left|N_{i}\left(y_{2}\right)\right|\right)=\sum_{i=1}^{k}\left(\left|Y_{i 1}\right|+\left|Y_{i 2}\right|\right) \\
& =\sum_{i=1}^{k}\left|Y_{i 1} \cup Y_{i 2}\right| \leq|T|-k=n-2 k-1 .
\end{aligned}
$$

On the other hand, we have

$$
\frac{1}{2} n \leq\left|N(x) \cup N\left(y_{i}\right)\right|=|N(x)|+\left|N_{T}\left(y_{i}\right)\right|=k+\left|N_{T}\left(y_{i}\right)\right| \quad(i=1,2),
$$

whence $\left|N_{T}\left(y_{1}\right)\right|+\left|N_{T}\left(y_{2}\right)\right| \geq n-2 k$, contradicting (12). 


\section{References}

[1] D. Bauer, G. Fan, and H. J. Veldman, Hamiltonian properties of graphs with large neighborhood unions. Preprint (1989).

[2] J. A. Bondy, Longest paths and cycles in graphs of high degree. Research report CORR80-16, Department of Combinatorics and Optimization, University of Waterloo, Waterloo, Ontario, Canada (1980).

[3] J.A. Bondy and U.S.R. Murty, Graph Theory with Applications. Macmillan, London, and Elsevier, New York (1976).

[4] R. J. Faudree, R. J. Gould, M. S. Jacobson, and L. M. Lesniak, Neighborhood unions and a generalization of Dirac's Theorem. Preprint (1988).

[5] R. J. Faudree, R. J. Gould, M.S. Jacobson, and L. M. Lesniak, Neighborhood unions and highly hamiltonian graphs. Preprint (1988).

[6] R. J. Faudree, R. J. Gould, M. S. Jacobson, and R. H. Schelp, Neighborhood unions and hamiltonian properties in graphs. J. Combinat. Theory B 46 (1989) 1-20.

[7] O. Ore, Note on Hamilton circuits. Am. Math. Month. 67 (1960) 55.

[8] H. J. Veldman, Existence of dominating cycles and paths. Discrete Math. 43 (1983) 281-296. 